\title{
HI observations of the starburst galaxy NGC 2146
}

\author{
A. Taramopoulos ${ }^{1,2}$, H. Payne ${ }^{3}$, and F. H. Briggs ${ }^{4}$ \\ 1 Technological Institute of Thessaloniki, Department of Electronics, Al. Papanastasiou 13, Thessaloniki 54639, \\ Greece \\ 2 Library Information Services, University of Macedonia, Egnatia 156, Thessaloniki 54006, Greece \\ e-mail: ttar@uom.gr \\ 3 Space Telescope Science Institute, 3700 San Martin Drive, Baltimore, MD 21218, USA \\ e-mail: payne@stsci.edu \\ 4 Kapteyn Astronomical Institute, Postbus 800, 9700 AV Groningen, The Netherlands \\ e-mail: fbriggs@astro.rug.nl
}

Received 18 May 2000 / Accepted 16 October 2000

\begin{abstract}
NGC 2146 is a peculiar spiral galaxy which is currently undergoing a major burst of star formation and is immersed in a extended HI structure that has morphological and kinematical resemblence to a strong tidal interaction. This paper reports aperture synthesis observations carried out in the $21 \mathrm{~cm}$ line with the Very Large Array $\left(\mathrm{VLA}^{\star}\right)$ of two fields positioned to optimally cover the H I streams to the north and south of the galaxy, along with a $300 \mathrm{ft}$ total power spectral mapping program to recover the low surface brightness extended emission. The observations reveal elongated streams of neutral hydrogen towards both the north and the south of the optical galaxy extending out up to 6 Holmberg radii. The streams are not in the principle plane of rotation of the galaxy, but instead are suggestive of a tidal interaction between NGC 2146 and a LSB companion that was destroyed by the encounter and remains undetected at optical wavelengths. Part of the southern stream is turning back to fall into the main galaxy, where it will create a long-lived warp in the H I disk of NGC 2146. Analysis of the trajectory of the outlying gas suggests that the closest encounter took place about 0.8 billion years ago and that infall of debris will continue for a similar time span.
\end{abstract}

Key words. galaxies: individual: NGC 2146 - galaxies: interactions - galaxies: kinematics and dynamics - galaxies: spiral - gadio lines: galaxies

\section{Introduction}

NGC 2146 is a peculiar spiral galaxy as seen from both the optical image and the HI line profile. Although it is classified as Sab by de Vaucouleurs et al. (1976), it shows a broad range of peculiarities. Measured by its far infrared flux, it is one of the 12 brightest galaxies in the sky and lies at a distance of $12.2 \mathrm{Mpc}\left(H_{0}=75 \mathrm{~km} \mathrm{~s}^{-1} \mathrm{Mpc}^{-1}\right.$, and $1^{\prime}$ corresponds to $3.5 \mathrm{kpc}$ ). In optical images there are two well-defined arms which mark the principal plane of rotation. Superimposed across part of the nucleus is an absorption band having "the form of a hand, with four talonlike fingers" (Pease 1920), which de Vaucouleurs (1950) interpreted as being a third arm inclined to the plane of rotation. Further optical studies by Benvenuti et al. (1975), however, suggest that a simple spiral model is not adequate.

Send offprint requests to: A. Taramopoulos

* The National Radio Astronomy Observatory (NRAO) is operated by Associated Universities, Inc. under cooperative agreement with the National Science Foundation.
In 1976, Fisher and Tully mapped the region around this galaxy in the $21 \mathrm{~cm}$ using the NRAO 91-m telescope with a resolution of $11 ! 3 \times 10 ! 2$ in the N-S and E-W directions respectively and discovered an extensive "envelope" of neutral hydrogen around it which extends up to six Holmberg radii $(100 \mathrm{Kpc})$. They suggest that the abnormalities seen both optically and in the neutral hydrogen profile of the main disk are probably related to the large H I extensions observed, and believe that they might be the result of
a) tidal interaction;
b) explosion/ejection, or;
c) galaxy formation.

They rule out any form of interaction between NGC 2146 and NGC 2146A, an Sc typed galaxy with no evident optical abnormalities, which lies about 20 arcsec away and is redshifted $595 \mathrm{~km} \mathrm{~s}^{-1}$ with respect to NGC 2146 . However, their observations mainly aimed to trace the total extent of the HI cloud, and their resolution was not good enough to allow them to draw more definite conclusions on 
the causes of these abnormalities. Also, the appearance of the H I envelope as a large gaseous halo around the main galaxy left room for speculations as to how large galactic halos really are, and what the impact of this might be on the QSO absorption line system statistics.

NGC 2146 contains a strong radio source, $\sim 3 \mathrm{kpc}$ in size, within its nuclear region, identified with 4C 78.06 (Caswell \& Wills 1967). Kronberg \& Biermann (1981) used the NRAO interferometer and the VLA to map the radio structure of the nuclear region. They found that the radio center lies in the optically obscured dust lane, but it shows no evidence of a double nucleus. The radio continuum map agrees very well with the CO intensity map, and unlike the optical image, it shows a remarkable degree of symmetry (Kronberg \& Biermann 1981; Jackson \& Ho 1988). The velocity curves measured in various lines in the optical and infrared are quite regular after allowance for extinction effects due to the dust lane (Prada et al. 1994). Kronberg \& Biermann (1981) suggested that a strong burst of star formation is responsible for the strong radio and infrared emission. A ${ }^{12} \mathrm{CO},{ }^{13} \mathrm{CO}$ and $\mathrm{CS}$ study undertaken by Xie et al. (1994) places the average temperature in the nuclear region about $55 \mathrm{~K}$, and the average density at $210^{4} \mathrm{~cm}^{-3}$. Further evidence of a burst of start formation comes from X-ray observations carried out by Armus et al. (1995) and Della Ceca et al. (1999) which reveal a galactic-scale outflow of gas driven by an intensive star bursting activity, referred to as a starburst-driven superwind. Furthermore, this activity can produce long-lived bending instabilities as suggested by $N$-body simulations carried out by Griv \& Chiueh (1998) to explain the snakeshaped radio structure observed by Zhao et al. (1996) at an angular resolution of $2^{\prime \prime}$ using the VLA.

Observations probing the molecular content in $\mathrm{CO}$ and $\mathrm{H}_{2}$ as well as the ionized gas content (H II regions) were made by Young et al. (1988). They found a very large concentration of gas in the nucleus, confirming Condon et al. (1982) earlier conclusion that this galaxy has a high star-formation rate and then, derived a mass-to-light ratio characteristic of very young stellar systems. All the above led these authors to suggest that NGC 2146 has recently undergone a collision with some other galaxy. The existance of an extended arc of H II regions encircling the central bright region, which exhibit velocities which are $130-200 \mathrm{~km} \mathrm{~s}^{-1}$ higher than those expected if they are rotating in the plane of the galaxy (Young et al. 1988), might also be interpreted as evidence of a collision.

After undertaking an optical and infrared study of the galaxy, Hutchings et al. (1990) found no sign of an active nucleus but did find many signs of a significant population of hot young stars in the central regions of the system. They concluded that NGC 2146 is a merging system, now in its final stages. The dominant galaxy is seen close to edge on, and the small companion has been stripped, leaving no sign of its nucleus. They also note that such a scenario can also be supported by numerical simulations (Barnes 1990).
In order to better understand the nature of this system and decide amongst the various scenaria which have been suggested, we obtained higher resolution $21 \mathrm{~cm}$ maps of the $\mathrm{H}$ I distribution around NGC 2146 using the VLA, and combined these with $21 \mathrm{~cm}$ observations of the NRAO 91-m telescope to recover the emission on large angular scales, which the interferometric observations alone are incapable of sensing. The nature of large gaseous halos is important in the interpretation of QSO absorption line spectra (cf. Rao \& Briggs 1993), where the large cross sections implied by the Fisher \& Tully observations (1976) would cause this kind of galaxies to intervene frequently by chance if they are common in the galaxy population.

\section{Observations and reduction}

The observations presented here consist of two parts. Interferometry observations, carried out with the Very Large Array, and single dish observations, carried out with the $300 \mathrm{ft}$ NRAO antenna before it collapsed.

On 25, 26 and 29 October 1984, the VLA was used in its most compact configuration to observe two fields, centered at $\alpha_{1950}=6^{\mathrm{h}} 12^{\mathrm{m}} 00^{\mathrm{s}}, \delta_{1950}=+78^{\circ} 10^{\prime} 00^{\prime \prime}$ and at $\alpha_{1950}=6^{\mathrm{h}} 10^{\mathrm{m}} 00^{\mathrm{s}}, \delta_{1950}=+78^{\circ} 35^{\prime} 00^{\prime \prime}$ respectively. The center fields were chosen in such a way that their combination produces a nearly uniform response over the area between and including the two pointing coordinates; this covers the main body of the galaxy and substantial fraction of the extended H I emission. A total of 5.5 hours per field with 25 antennas with baselines ranging from $36 \mathrm{~m}$ to a little more than $1 \mathrm{~km}$ were used. We observed only the right polarization in a band centered at a heliocentric velocity of $915 \mathrm{~km} \mathrm{~s}^{-1}$. After on-line Hanning smoothing, we recorded 31 independent channels ranging from 583 to $1226 \mathrm{~km} \mathrm{~s}^{-1}$ with a FWHM velocity resolution of $20.75 \mathrm{~km} \mathrm{~s}^{-1}$. A broader bandwidth "continuum channel 0 " to accompany the line data was calculated on-line from the average of 48 such channels centered at $915 \mathrm{~km} \mathrm{~s}^{-1}$. The VLA observational parameters are summarized in Table 1. For the flux calibration we used 3C 286, adopting a flux density of 14.88 Jy according to the latest VLA measurements. The phase calibration source was $0836+710$ for which a flux density of $4.12 \mathrm{Jy}$ at $1.416 \mathrm{MHz}$ was determined. Both calibrators were used in extracting a measure of the shape of the passband which we applied as a correction to the observations of NGC 2146.

To optimize the sensitivity to weak extended H I emission, the Fourier transformation was carried out using a natural weighting for the data, i.e. each visibility cell in the $u-v$ plane has been weighted according to the integration time spent on it. This gives the best signal-tonoise ratio for detecting weak sources. Since $u-v$ tracks tend to spend more time per unit area near the $u-v$ origin, natural weighting emphasizes the data from short spacings, and produces a broad synthesized beam with an extended, low-level sidelobe. The natural-weighted maps provide sensitive information on the larger scale and are suitable for analyzing the structure of the extended H I 
Table 1. The VLA observational parameters

\begin{tabular}{ll}
\hline \hline Instrument & Very Large Array (VLA) \\
Object & NGC 2146 \\
Total observing time & 11 hours \\
Pointing center & \\
South field RA (1950.0) & $6^{\mathrm{h}} 12^{\mathrm{m}} 00^{\mathrm{s}}$ \\
\multicolumn{1}{c}{ dec. $(1950.0)$} & $+78^{\circ} 10^{\prime} 00^{\prime \prime}$ \\
North field RA $(1950.0)$ & $6^{\mathrm{h}} 10^{\mathrm{m}} 00^{\mathrm{s}}$ \\
$\quad$ dec. (1950.0) & $+78^{\circ} 35^{\prime} 00^{\prime \prime}$ \\
Flux density calibrator & $3 \mathrm{C} 286$ \\
Phase calibrator & $0836+710$ \\
Central velocity of spectral band & $915 \mathrm{~km} \mathrm{~s}^{-1}$ \\
Configuration & $\mathrm{D} \mathrm{Array}$ \\
Observed baselines (min-max) & $40-1030 \mathrm{~m}^{\prime}$ \\
Number of antennas & 25 \\
Number of channels & 31 \\
Channel spacing & $20.75 \mathrm{~km} \mathrm{~s}^{-1}$ \\
FWHM velocity resolution & \\
Total line bandwidth & $20.75 \mathrm{~km} \mathrm{~s}^{-1}$ \\
& $3 \mathrm{MHz}$ \\
Total continuum bandwidth & $643.5 \mathrm{~km} \mathrm{~s}^{-1}$ \\
Type of weighting of $u-v$ data & $4.69 \mathrm{MHz}$ \\
R.m.s. noise per channel & $1-1.25 \mathrm{mJy}^{\prime \prime}$ \\
Synthesized Beam (N-S E-W) & $49^{\prime \prime} \times 77^{\prime \prime}$ \\
\hline \hline
\end{tabular}

Note: $a$. On-line Hanning taper mode.

cloud of NGC 2146. However, the poor synthesized beam shape means that care must be taken in the deconvolution phase of the data processing. Incorporation of the single dish spectral is required for reliable mapping of the extended structure.

The construction of the continuum subtracted channel maps for each of the two fields is a crucial step in the reduction. Due to restrictions in the number of spectral channels that could be recorded in 1986 by the VLA, the correlator configuration was chosen so that it had H I emission in 29 of the 31 velocity channels. The on-line "channel 0", being a mean of 48 channels, 29 of which are corrupted by the strong H I emission does not represent a true continuum channel. A true continuum map was obtained according to the following scheme, which is a weighted difference between spectral channel maps with gas and the "channel 0" map:

Cont.Map $=\frac{1}{(48-31)}\left(48(\right.$ Ch. 0$)-\sum_{i=1}^{31}$ Ch. $\left.i\right)$.

In this way, the continuum map was formed from the frequency band outside the channels containing H I emission. The continuum was then subtracted from the dirty line channel maps to obtain the narrow band HI emission. This yielded a continuum field of total flux $1.83 \mathrm{Jy}$ with the brightest source being the galaxy NGC 2146 (0.75 Jy).

During September 1987 the $300 \mathrm{ft}$ NRAO telescope was used to observe the area of NGC 2146. On each day a single drift scan was performed at a fixed declination, and spectra were recorded in short integrations. At the end of the observing session, a critically sampled map of the region surrounding NGC 2146 for merging with the interferometer data was constructed by gridding the data onto the same spatial and velocity grids as used for the VLA data cubes. For the joint deconvolution performed simultaneously on the VLA and $300 \mathrm{ft}$ data, a Gaussian beam of HPBW 11.3 arcmin in the direction N-S and 10.2 arcmin in the direction E-W was adopted for the $300 \mathrm{ft}$ (Fisher \& Tully 1975).

The continuum-free VLA maps were then simultaneously cleaned, mosaiced and combined together with the single dish maps with the AIPS maximum entropy based task UTESS to form single maps for each channel. At this stage, tests were performed using other alternative approaches of deconvolution like CLEAN algorithms alone, or combinations of CLEAN and maximum entropy based algorithms (implemented by VTESS in AIPS), yielding similar results in terms of map quality, but making apparent the fact that the single dish data were adding to the final maps substantial flux that the interferometry maps alone were missing, due to the lack of short spacing $u-v$ coverage.

The final natural-weighted channel maps have an rms noise per channel of 1.0 to $1.25 \mathrm{mJy} / \mathrm{beam}$, which is approximately the theoretical value $(1.0 \mathrm{mJy} / \mathrm{beam})$. The final synthesized beam is $49^{\prime \prime} \times 77^{\prime \prime}$ in the North-South and East-West directions respectively.

\section{The $\mathrm{HI}$ distribution}

The channel maps of the H I flux of the area around the optical galaxy are shown in Figs. 1 and 2. The channels containing emission at large angular distance from the galaxy, together with a map of the radio continuum at $1420 \mathrm{MHz}$, appear in Figs. 3 and 4. These maps reveal elongated streams of neutral hydrogen towards the north and the south of the main galaxy, extending out up to 6 Holmberg radii. This extensive distribution of the H I has a "tail" morphology suggestive of a tidal interaction of NGC 2146 and a companion, which has not been identified in optical images.

The southern stream is detected at higher signal to noise ratio than the northern stream. This occurs because the southern gas appears in only a few velocity channels, where the spatial structure is dominated by a single long arc that is nearly coincident in the three main contributing channels. There are extensions to this arcing stream, which, instead of following the general outflow of the HI, lie in smaller arcs of gas that appear to turn eastward and separate from the stream, falling back toward the central potential of the system. Further out from the tip of the southern stream appear some small H I clouds, which do not seem to be gravitationally bound to the whole system and may be escaping towards the south east.

The stream at the north is less prominent, due in part to being more heavily resolved, both spatially and in velocity, than the southern stream. Unlike the nearly constant velocity measured in the south, there is a prominent 


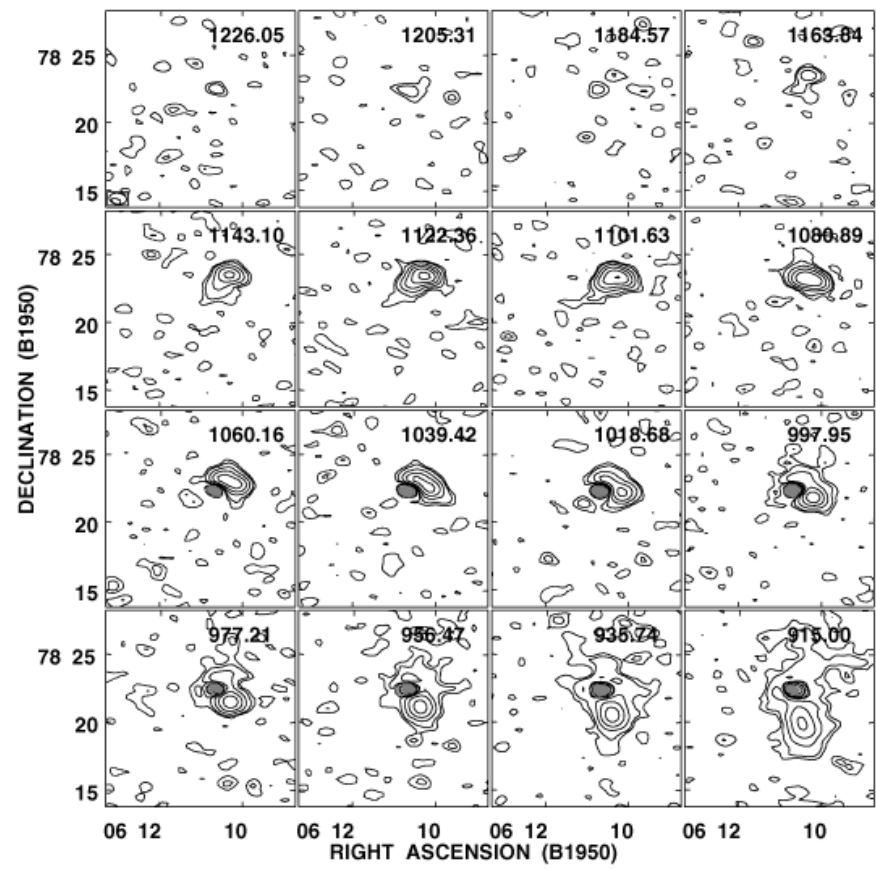

Fig. 1. The first 16 spectral line channel maps of H I emission with $20.75 \mathrm{~km} \mathrm{~s}^{-1}$ wide channels for the central area around NGC 2146. The numbers appearing on the upper right corner of each channel map are the velocities of the H I in $\mathrm{km} \mathrm{s}^{-1}$. The grey areas correspond to $\mathrm{HI}$ absorption. Contours are drawn at $-10,-3.5,-2,2,3.5,8,15,25$ and $40 \mathrm{mJy}$. The peak flux is $46.3 \mathrm{mJy}$

velocity gradient across the northern extended emission from $\sim 975 \mathrm{~km} \mathrm{~s}^{-1}$ at position angle $-15^{\circ}$ to $\sim 850 \mathrm{~km} \mathrm{~s}^{-1}$ at position angle $\sim 30^{\circ}$. Thus, the northern stream is symmetrically placed with respect to the southern one, but is not its mirror image. Instead of being concentrated in only three of our channels $\left(\sim 60 \mathrm{~km} \mathrm{~s}^{-1}\right)$, the northern stream appears to be spread in a fan shaped outflow with line-ofsight velocity components ranging from $\sim 60 \mathrm{~km} \mathrm{~s}^{-1}$ redshifted to $\sim 65 \mathrm{~km} \mathrm{~s}^{-1}$ blueshifted as a function of position angle on the sky.

The observed HI distribution is consistent with that found by Fisher \& Tully (1976), given the $10^{\prime}$ beam (FWHM) of the 91-m NRAO telescope. One significant difference is that the VLA detects absorption against the position of the nuclear continuum emission of the galaxy. This absorption feature persisted throughout all our reduction, and was consistently present in both the VLA maps of the North and the South part of NGC 2146, although the position of the galaxy was at a different point in the primary beam for each case. Also, the fact that no deep bowls were seen around the H I distribution suggests that the absorption is real and not an artifact of the interferometer data reduction. The spectrum of the absorption is shown in Fig. 5 with 5- $\sigma$ errorbars plotted for each spectral channel. This absorption feature would certainly be missed by a single dish telescope because of its large beam, in which the absorption is diluted and overwhelmed by the

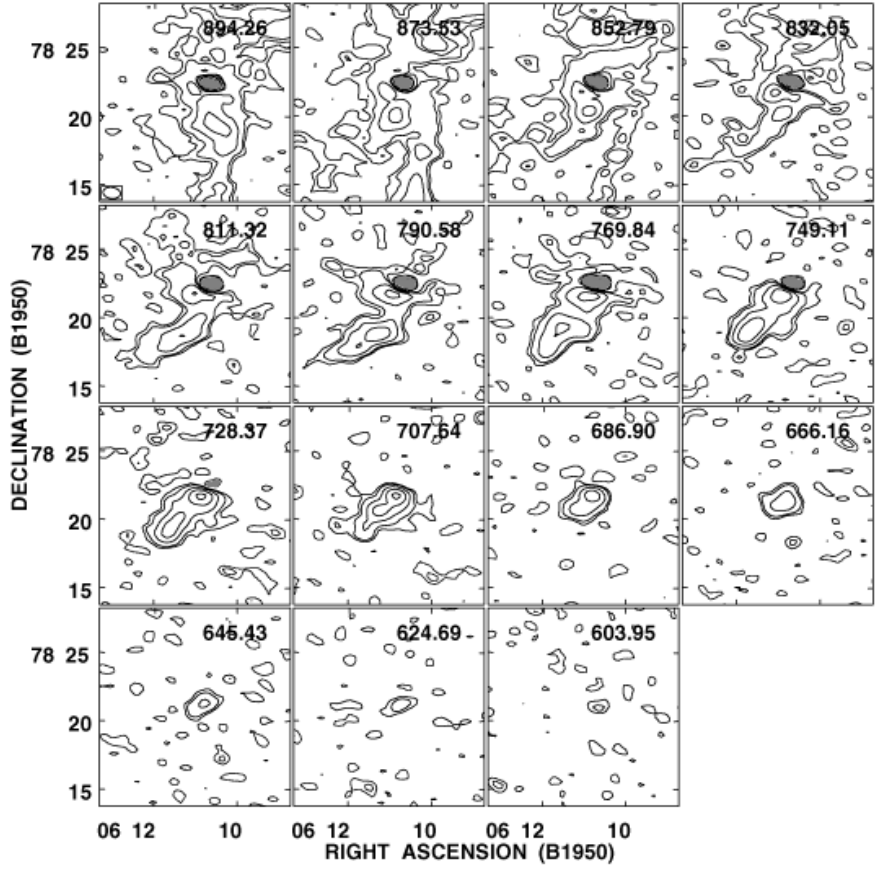

Fig. 2. The last 15 spectral line channel maps of H I emission with $20.75 \mathrm{~km} \mathrm{~s}^{-1}$ wide channels for the central area around NGC 2146. The numbers appearing on the upper right corner of each channel map are the velocities of the $\mathrm{HI}$ in $\mathrm{km} \mathrm{s}^{-1}$. The grey areas correspond to HI absorption. Contours are drawn at $-10,-3.5,-2,2,3.5,8,15,25$ and $40 \mathrm{mJy}$. The peak flux is $46.3 \mathrm{mJy}$

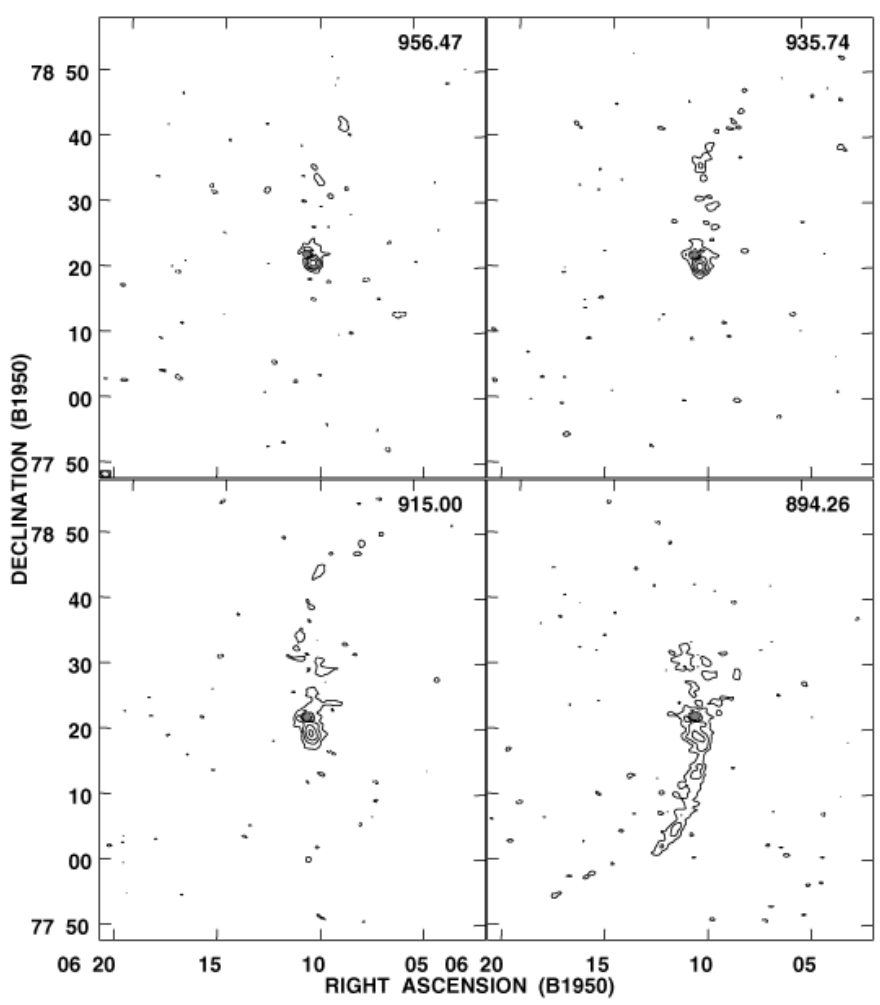

Fig. 3. H I spectral line channel maps showing the extended streams at the north and south parts of NGC 2146. The grey areas correspond to $\mathrm{H}$ I absorption. Contours are drawn at -10 , $-3.5,3.5,8,15,25$ and $40 \mathrm{mJy}$ 


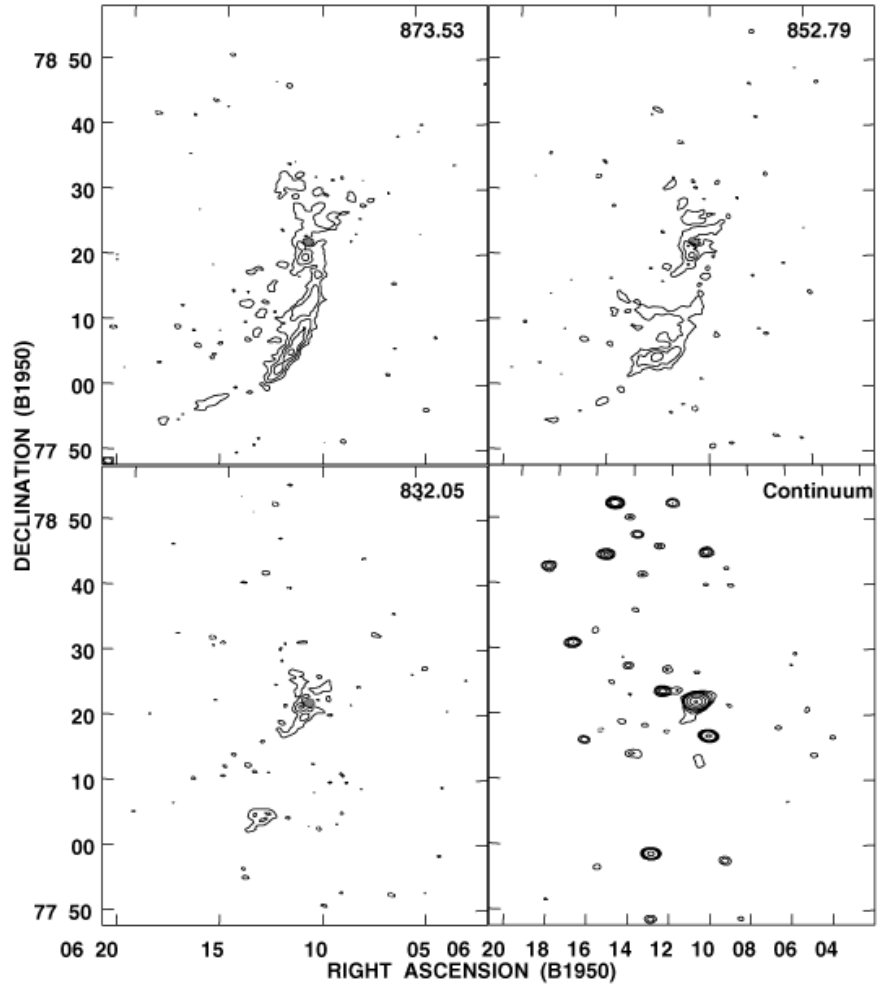

Fig. 4. H I Spectral line channel maps showing the extended streams at the north and south parts of NGC 2146 and the continuum map of the area. The grey areas correspond to H I absorption. Contours for the channel maps are drawn at -10 , $-3.5,3.5,8,15,25$ and $40 \mathrm{mJy}$. For the continuum the contours are drawn at $2,4,8,16,64,256$ and $700 \mathrm{mJy}$, with the peak flux being $755 \mathrm{mJy}$

integrated emission. Figure 5 also shows the spectrum of NGC 2146 obtained by Fisher \& Tully (1976) by pointing the 91-m telescope directly at the center of NGC 2146 (dot-dashed line), the spectrum of the whole area containing the H I cloud made by adding up all the flux seen by the NRAO 91-m telescope (solid line), and the spectrum of the total H I obtained after combining the VLA data with those of the NRAO 91-m telescope (solid line with $\mathrm{Xs}$ ). Apparently, we are still missing about $20 \%$ of the total H I flux; maps produced from the VLA data alone were missing about $50 \%$ before being combined with the single dish data. This deconvolution procedure was successful in bringing up some fine details in the faint extended emission, especially in the northern stream of gas, where the signal to noise ratio is low due to being heavily resolved, and also in the low level emission to the east of the southern arm.

The absorption is seen against the radio center of the galaxy, which lies in the region of the optically obscuring dust, and is unresolved by our observations. It has a velocity width of $\sim 350 \mathrm{kms}^{-1}$ and an average optical depth of 0.03. Assuming a spin temperature of $50 \mathrm{~K}$, the measured optical depth requires an H I column density of $\sim 10^{21}$ atoms $\mathrm{cm}^{-2}$. The absorption is centered at the galaxy's systemic velocity and seems to be due to H I
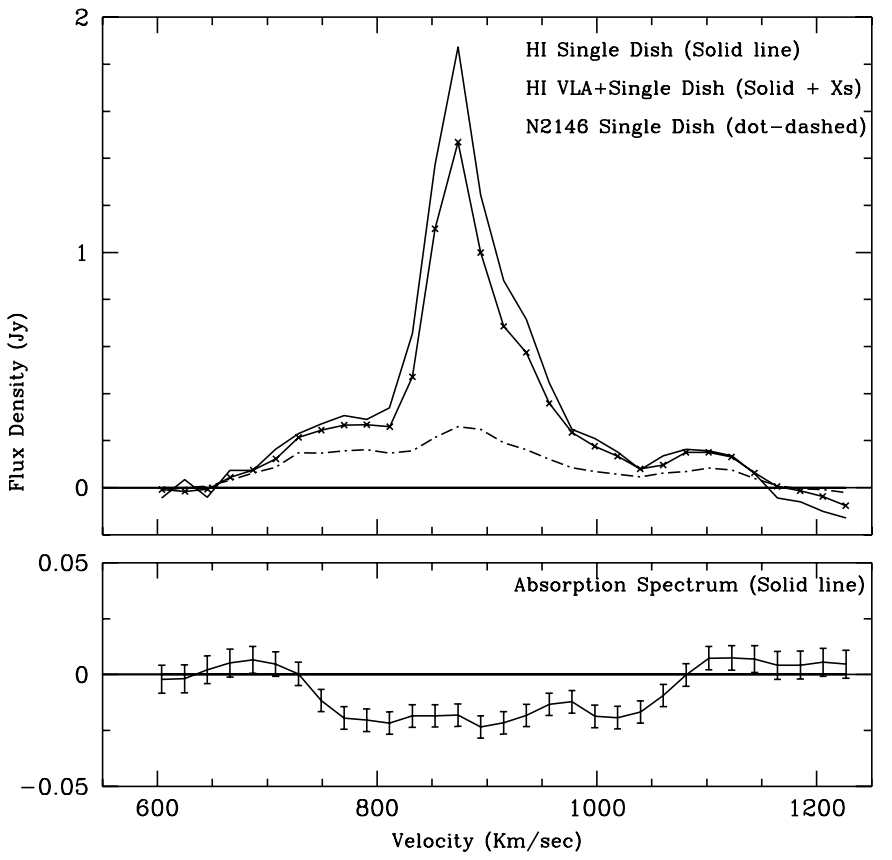

Fig. 5. The H I single dish spectrum of the whole area around NGC 2146, and the one obtained after deconvolving the single dish maps with the VLA ones to improve the sensitivity. Also is shown the NGC 2146 H I profile obtained by Fisher \& Tully (1975) by pointing the telescope to the optical image of the galaxy (dot dashed line). At the bottom is shown the spectrum of the absorption detected by the VLA against the radio continuum of NGC 2146 with $5-\sigma$ errorbars plotted for each spectral channel

clouds rotating together with the rest of the H I seen in emission, which happen to lie in front of the strong radio continuum source of NGC 2146, 4C 78.06. However, the large velocity gradient seen accross its $1^{\prime}$ span, compared to the total width of the H I profile of NGC 2146 $\left(\sim 500 \mathrm{~km} \mathrm{~s}^{-1}\right)$ implies that the H I absorbing layer is not very far from the nucleus of NGC 2146. If $V_{0}$ is the total H I profile velocity width of NGC $2146\left(\sim 500 \mathrm{~km} \mathrm{~s}^{-1}\right)$, and $\Delta v$ is the velocity width of the absorbing layer seen against the NGC 2146 continuum emission $\left(\sim 350 \mathrm{~km} \mathrm{~s}^{-1}\right)$ then, assuming a circularly rotating system

$\frac{\Delta v}{2}=\frac{V_{0}}{2} \sin \phi_{\mathrm{abs}}$

where $\phi_{\mathrm{abs}}$ is shown in Fig. 6 . From Fig. 6 we also see that

$\sin \phi_{\mathrm{abs}}=\frac{L / 2}{R}$

where $L$ is the length of the absorbing layer projected on the plane of the sky $(\sim 1$ arcsec, or $3.5 \mathrm{kpc}$; this is an upper limit imposed by the spatial resolution of our observations), and $R$ is its distance from the nucleus. Combining these two equations we estimate an upper limit for the distance of the absorbing layer from the continuum background to be

$R=\frac{L V_{0}}{2 \Delta v}=2.5 \mathrm{kpc}$. 


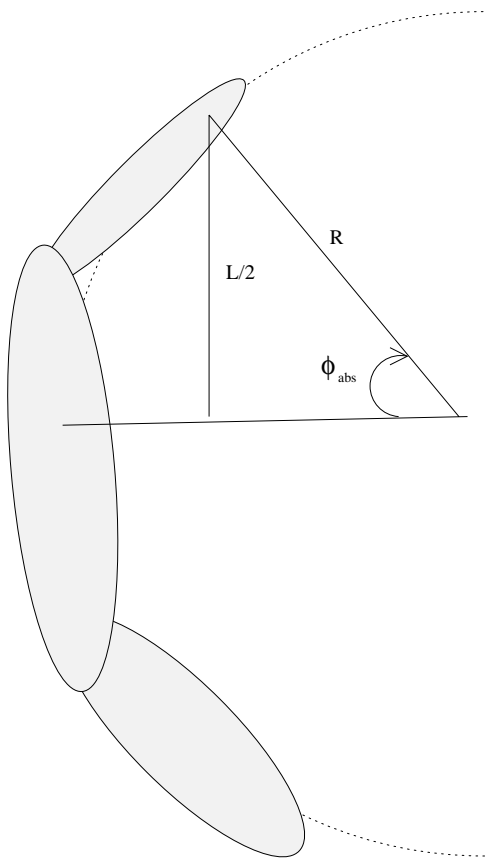

Fig. 6. Schematic diagram of the HI absorption layer in NGC 2146

The integrated HI flux density map is shown in Figs. 7 (large scale) and 8 (small scale). We estimate the total mass for the cloud to be about $6.210^{9} M_{\odot}\left(H_{0}=\right.$ $75 \mathrm{~km} \mathrm{~s}^{-1} \mathrm{Mpc}^{-1}$ ) of which $1.610^{9} M_{\odot}$ come from the bright region around the galaxy itself, and $4.610^{9} M_{\odot}$ from the extended component. Furthermore, the extended distribution is not symmetric with respect to the amount of gas in the south $\left(\begin{array}{llll}3.1 & 10^{9} M_{\odot}\end{array}\right)$ and the north $\left(1.510^{9} M_{\odot}\right)$. For the above calculations a velocity component of $223 \mathrm{~km} \mathrm{~s}^{-1}$ of our Sun towards NGC 2146's direction has been assumed, in order to refer the redshift velocity to the center of mass of the Local Group.

Figures 9 and 10 show the intensity weighted mean radial velocity field on both large and small scale. Points with negative flux due to absorption have been excluded from this calculation, and only emission brighter than $1.3 \mathrm{mJy}$ has been taken into account. It is clear from the velocity field that the main galaxy has a differentially rotating disk with characteristic rotational speed of about $250 \mathrm{~km} \mathrm{~s}^{-1}$. The extended H I disk, however, appears severly lopsided as can immediately be seen from Fig. 10 since the galaxy is seen almost edge on.

The HI disk of the main galaxy has a well defined plane of rotation, with an inclination nearly perpendicular to the sky plane. The extended HI stream to the south, however, has very little velocity gradient along its entire length, with an average velocity that is coincident with the systemic velocity of the central galaxy. This situation occurs when the outlying stream and the galaxy centroid lie in the plane of the sky and implies that we observe the southern stream from a preferred viewing angle, in which it appears laid out in the probable plane of the interaction, which must have been very nearly

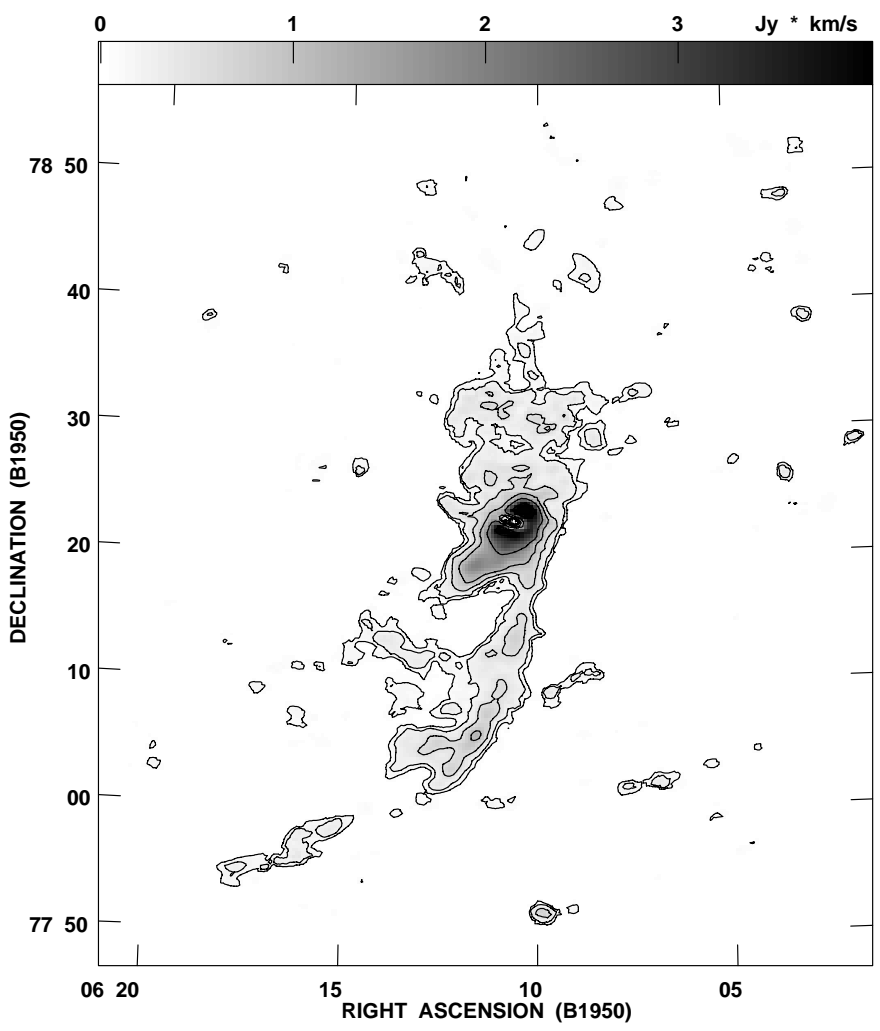

Fig. 7. The total integrated H I flux density of NGC 2146. Contours are drawn at $-0.5,-0.2,-0.1,0.1,0.2,0.5,1,2,4$ and $5 \mathrm{Jy} \mathrm{km} \mathrm{s}^{-1}$. The peak flux is $5.8 \mathrm{Jy} \mathrm{km} \mathrm{s}^{-1}$

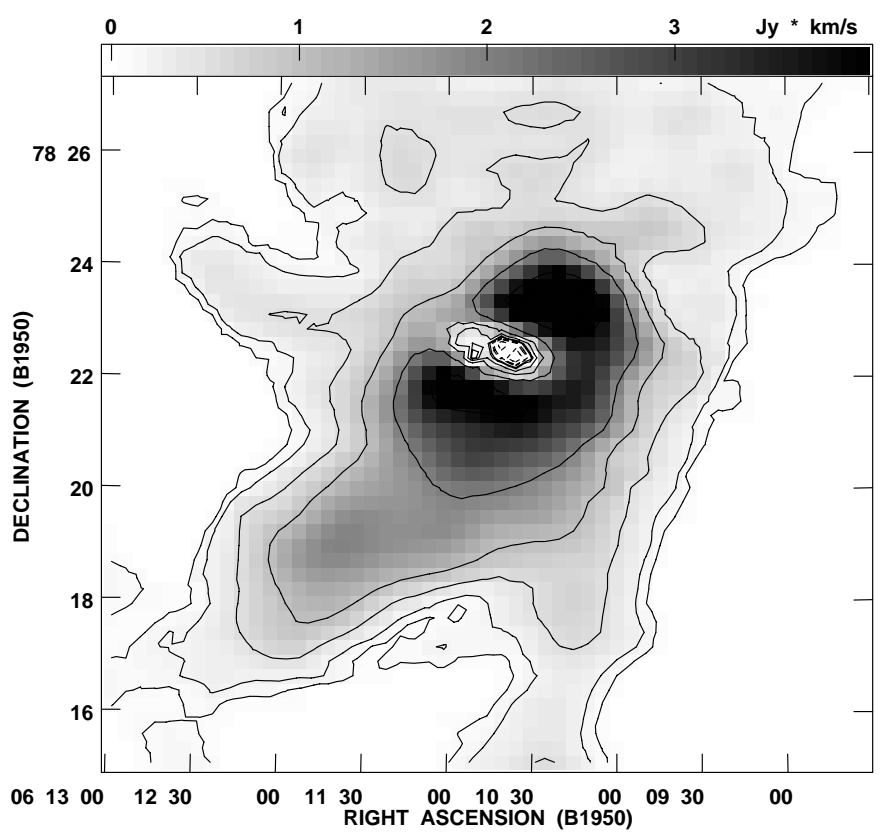

Fig. 8. The total integrated HI flux density of NGC 2146. Contours are drawn at $-0.5,-0.2,-0.1,0.1,0.2,0.5,1,2,4$ and $5 \mathrm{Jy} \mathrm{km} \mathrm{s}^{-1}$. The peak flux is $5.8 \mathrm{Jy} \mathrm{km} \mathrm{s}^{-1}$

perpendicular to the disk plane of NGC 2146. The northern stream, on the other hand, does show a significant velocity gradient across it, and the orientation of the stream cannot be inferred in a model-independent way. 


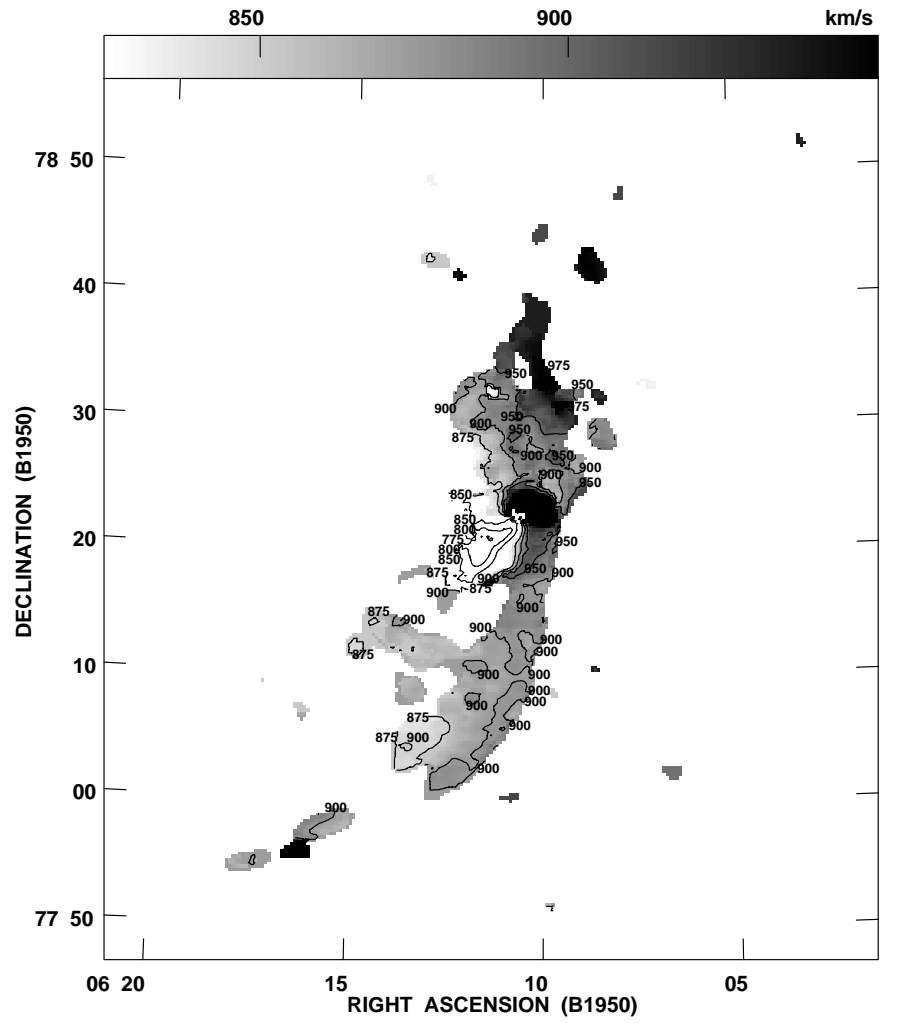

Fig. 9. The H I intensity weighted mean radial velocity field of NGC 2146. Contours are drawn at 750, 775, 800, 850, 875, 900, $950,975,1000,1050$ and $1100 \mathrm{~km} \mathrm{~s}^{-1}$. The greatest velocity is $1201 \mathrm{~km} \mathrm{~s}^{-1}$

In Fig. 11 the total integrated HI flux density of NGC 2146 is plotted superimposed on an optical image of the galaxy from the Digitized Sky Survey (DSS1). The morphology of the HI cloud does not show any preferential alignment with respect to the direction of NGC 2146A, and therefore shows no evidence for interaction between NGC 2146 and NGC 2146A. The faint arm extending southwards from the top NW corner of the optical image is in the general direction of the H I southern stream. However, the velocity gradient of H II regions of $\sim 100 \mathrm{~km} \mathrm{~s}^{-1}$ observed by Young et al. (1988) across this feature is incompatible with the absence of a velocity gradient in the southern H I stream and its alignment with the plane of the sky. It seems thus that this arm is part of the main galaxy which was disturbed from an interaction but still remained under the strong gravitational influence of NGC 2146. The loop structure at the SE of the optical image of the galaxy shows higher velocities than those expected if it was rotating in the plane of the galaxy (Young et al. 1988) presenting further evidence for a tidal interaction. However, there is no apparent correspondence with any $\mathrm{HI}$ feature.

\section{Discussion}

The complex appearance of this system may represent the aftermath of an encounter between NGC 2146 and a slightly less massive but gas-rich galaxy, probably a late-

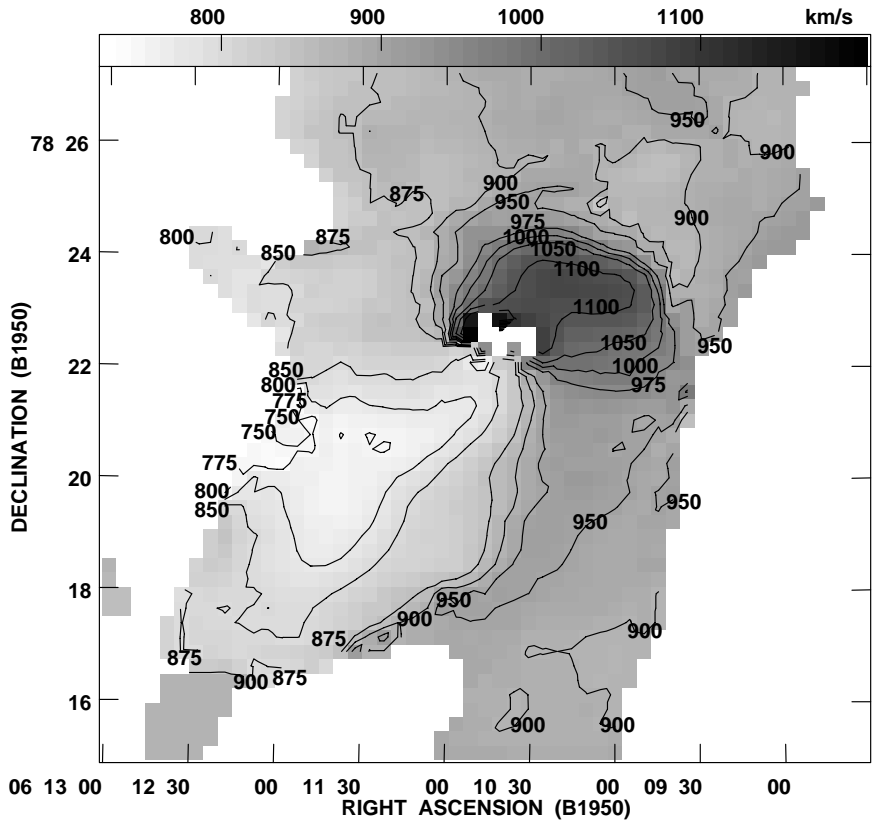

Fig. 10. The H I intensity weighted mean radial velocity field in the central region of NGC 2146. Contours are drawn at 750 , $775,800,850,875,900,950,975,1000,1050$ and $1100 \mathrm{~km} \mathrm{~s}^{-1}$. The greatest velocity is $1201 \mathrm{~km} \mathrm{~s}^{-1}$

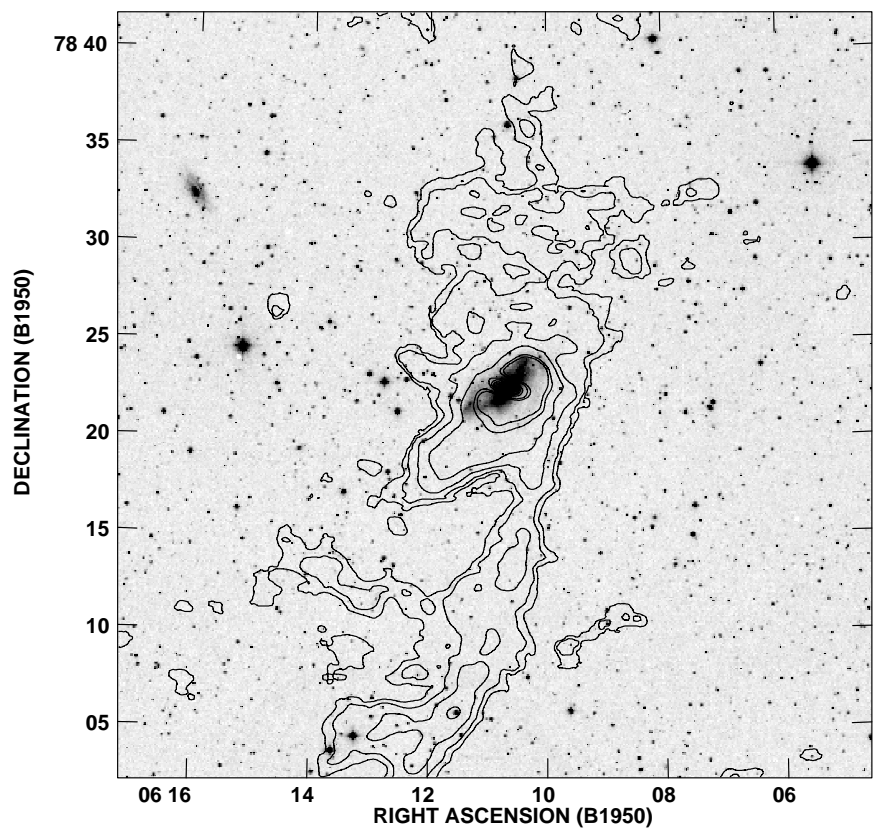

Fig. 11. The total integrated HI flux density of NGC 2146 superimposed on an optical image of the galaxy from the Digitized Sky Survey (DSS1). The contours drawn are the same as in Fig. 7

type LSB spiral, with a slowly rising rotation curve indicating little or no bulge (de Blok et al. 1996). Numerical simulations by Wallin \& Stuart (1992) indicate that the outcome of an interaction depends crucially on the orientation of each galaxy's rotational angular momentum vector relative to the plane of the interaction. If the rotational 


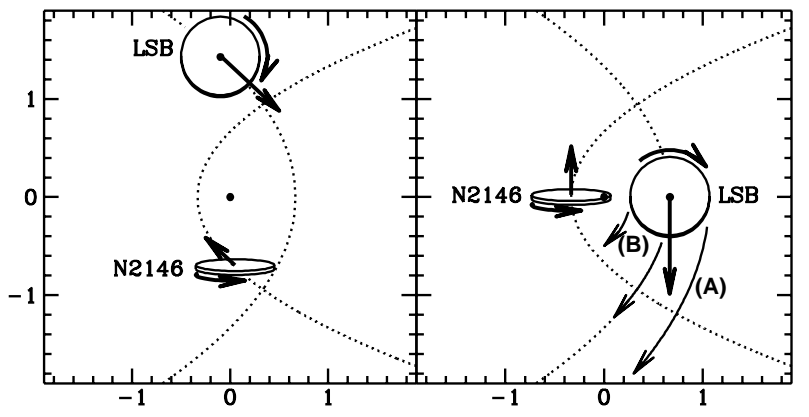

Fig. 12. Schematic of Interaction. Left Panel: Pre-encounter. Right Panel: Closest approach. The schematic shows the observer's view of an interacting system where the translation of the incident galaxies takes place in the sky plane. The rotational angular momentum vector for NGC 2146 galaxy lies in the sky plane, and the rotational angular momentum vector for an incident LSB galaxy is oriented perpendical to the sky plane

angular momentum vector lies in the plane of the interaction, the galaxy comes through with very little loss of mass. On the other hand, if the rotational angular momentum aligns with the orbital angular momentum, a large fraction of the galaxy is stripped away.

NGC 2146 may have interacted with a gas-rich "intruder" whose rotational angular momentum vector was oriented perpendicular to that of NGC 2146 and perpendicular to the plane of the interaction, which in this case is close to being coincident with the plane of the sky. Figure 12 is a schematic diagram of the encounter. In this configuration, NGC 2146 has a rotational angular momentum vector lying in the interaction plane and is able to preserve its identity as a rotating disk galaxy, while the same encounter essentially destroys the intruder.

The numerical simulations of Wallin \& Stuart (1992) predict in such cases that the mass fraction transfered from the intruder to the main galaxy can be as large as 0.5 , and the mass fraction lost by the intruder that eventually escapes from the whole system can reach up to 0.2 . Wallin \& Stuart used a model with restricted 3-body interactions (two large point masses binding massless test particles), and more realistic models with distributed mass are likely to suffer even greater destruction for the case of aligned orbital and rotational angular momentum, as is indicated by some test cases run for us by Gerritsen (private communication) using his tree-code implementation (Gerritsen 1997).

The outcome of the tidal interactions is the development of gas streams on opposite sides of both galaxies. The velocity gradient of the gas in the northern stream is consistent with the sense of rotation of NGC 2146, which may indicate that this gas originated from the $\mathrm{HI}$ disk of NGC 2146. The gas at the southern stream, however, appears only around the systemic velocity of the system, and is likely to be the tidally dispersed remnant of the intruder. As the galaxies swirled around each other, H I clouds released from the intruder's side opposite to NGC 2146 were boosted to escape velocity by the combination of the intruder's translational and rotational velocities. In this scenario, the outermost clouds of the intruder experience the gravitational slingshot effect familiar in interplanetary spacecraft trajectories to reach the outer solar system. We next test the plausibility of such an encounter by exploring the timing arguments for the formation and lifetime of the southern plume near NGC 2146. For our purposes, we approximate the relative speeds of the masses using the vis viva equation to describe the relative speed $V$ of the centers of mass for the two masses, $M_{\mathrm{NGC} 2146}$ and $M_{\mathrm{intr}}$, as a function of their separation $R$ and $a$, the semimajor axis of a possible elliptical orbit of the intruder about NGC 2146. Then,

$$
\begin{aligned}
V^{2} & =G\left(M_{\mathrm{NGC} 2146}+M_{\mathrm{intr}}\right)\left[\frac{2}{R}-\frac{1}{a}\right] \\
& =G M_{\mathrm{NGC} 2146}\left(1+\frac{M_{\mathrm{intr}}}{M_{\mathrm{N} 2146}}\right)\left[\frac{2}{R}\right] \\
& \approx\left(350 \mathrm{~km} \mathrm{~s}^{-1}\right)^{2}\left(1+\frac{M_{\mathrm{intr}}}{M_{\mathrm{NGC} 2146}}\right)\left[\frac{15 \mathrm{kpc}}{R}\right]
\end{aligned}
$$

where we have made the assumption that the intruder falls in from large distance so that $a \rightarrow \infty$ and that the mass of NGC 2146 can be approximated by adopting the rotational velocities of $V_{\mathrm{o}} \simeq 250 \mathrm{~km} \mathrm{~s}^{-1}$ that are measured in the outskirts of NGC 2146 at $R_{\mathrm{O}}=15 \mathrm{kpc}$ to obtain $G M_{\mathrm{NGC} 2146} \approx R_{\mathrm{o}} V_{\mathrm{o}}^{2}$.

For purposes of this illustration, we consider an HIrich, "LSB" intruder, with $M_{\text {intr }}=M_{\mathrm{NGC} \mathrm{2146}} / 4$ and $R_{\text {intr }} \approx R_{\mathrm{o}}$, having a gradually rising rotation curve that reaches $V_{\text {rot }} \approx 125 \mathrm{~km} \mathrm{~s}^{-1}$ at the outer edge of the galaxy's disk. Under these assumptions, the relative speeds of the centers of mass for the two galaxies will be $V \approx 277 \mathrm{~km} \mathrm{~s}^{-1}$ for a grazing encounter where $R=30 \mathrm{kpc}$. Four-fifths of the encounter speed $\left(\sim 222 \mathrm{~km} \mathrm{~s}^{-1}\right)$ is carried by the center of mass of this intruder at a distance of $24 \mathrm{kpc}$ from the system barycenter. By the time of closest approach, the intruder will have already become tidally distorted, and as it passes pericenter, simple tidal force arguments show that it will be unbound throughout its disk, provided it has a slowly rising rotation curve. However, in order to estimate the "launch speed" of debris in the outskirts of the intruder (trajectory A in Fig. 12), we consider the effect of adding the $V_{\text {rot }}$ of the intruder to the speed of its center of mass.

Under these assumptions, gas clouds at the far side of the intruder from NGC 2146 are traveling at $\sim 346 \mathrm{~km} \mathrm{~s}^{-1}$ at a distance of $39 \mathrm{kpc}$ from the barycenter. We estimate the escape velocity from this distance by lumping $M_{\mathrm{NGC} 2146}+M_{\text {intr }}$ at the barycenter to find $V_{\mathrm{esc}} \approx$ $240 \mathrm{~km} \mathrm{~s}^{-1}$, which is roughly $100 \mathrm{~km} \mathrm{~s}^{-1}$ less than the speed of the outer gas clouds. Once the process of dismantling the intruder begins, the mass lost lowers the binding energy, allowing the intruder to be shredded throughout.

Gas clouds on the inside of the intruder closest to the system barycenter, of course, find that whatever rotational component of velocity they still carry is counter to the 
orbit around NGC 2146. The dynamics in this region are clearly complicated, so that subtracting the $125 \mathrm{~km} \mathrm{~s}^{-1}$ rotational speed from the $222 \mathrm{~km} \mathrm{~s}^{-1}$ intruder center of mass speed to leave $\sim 100 \mathrm{~km} \mathrm{~s}^{-1}$ forms only a rough guess. However, the implication is still that the inner gas is progressing in the same direction as the bulk of the material from the dismemberment of the intruder.

As a result, we picture the southern HI plume to be the result of tidal shredding of a high angular momentum LSB galaxy. The test simulation by Gerritsen (private communication) confirms that this could be accomplished without enhancing star formation in the intruder.

In order to make some statement about the time span since closest approach, we make the following simplifying assumptions: first, the high speed clouds from the outskirts of the intruder escape (trajectory A in Fig. 12) from the system by overcoming the gravitation potential created by the the sum of the two original masses, concentrated at the system barycenter. Second, the remnants such as the HI spur running nearly east-west at declination $\sim 78^{\circ} 12^{\prime}$ find their acceleration dominated by the mass of NGC 2146 alone. If we assume that the gas in this spur is located at the turn-around point of its trajectory, we can estimate the major axis of its orbit and deduce the time span required for it to return to closest approach to NGC 2146. The spur falls $\sim 14^{\prime}$ or $49 \mathrm{kpc}$ from the center of NGC 2146. Assuming these clouds were launched from a distance of $\sim 25 \mathrm{kpc}$ on the opposite side of NGC 2146, this trajectory (B in Fig. 12) can be approximated by an ellipse of major axis $2 a=74 \mathrm{kpc}$. Using the rotational velocity $V_{\mathrm{o}}=\sim 250 \mathrm{~km} \mathrm{~s}^{-1}$ for NGC 2146 at $R_{\mathrm{o}}=15 \mathrm{kpc}$, we deduce that the orbital period $T(a)$ would be $(T / 0.4 \mathrm{Gyr})^{2}=(a / 15 \mathrm{kpc})^{3}$ as a function of $a$. Thus, half of the period for $a=37 \mathrm{kpc}$ is $\sim 0.8$ Gyr. This forms an estimate of the time elapsed since the closest approach.

The gas clouds ejected along trajectory A have reached $\sim 50^{\prime}$ or $180 \mathrm{kpc}$ during $0.8 \mathrm{Gyr}$, for an average speed of $225 \mathrm{~km} \mathrm{~s}^{-1}$. If the clouds were launched with the $346 \mathrm{~km} \mathrm{~s}^{-1}$ estimated above, then they will have decelerated along their path, consistent with this estimate for average speed.

In this scenario, the central regions of the destroyed intruder are likely to lie somewhere along the south stream. Indeed, there is a concentration of gas, located at declination $78^{\circ} 04^{\prime}$, where the south stream has a kink (most clearly seen in channels with velocities of 853 and $832 \mathrm{~km} \mathrm{~s}^{-1}$ in Fig. 4). The concentration has galaxy sized dimensions and a velocity gradient of $60 \mathrm{~km} \mathrm{~s}^{-1}$ across its $30 \mathrm{kpc}$ extent, contains $\sim 1.510^{8} M_{\odot}$ in neutral hydrogen, and has an HI column density of $\sim 210^{20}$ atoms $\mathrm{cm}^{-2}$, which is high enough to trigger star formation that might be detectable in deep CCD images. All these make this object a very good candidate of being a remnant of the long sought companion of NGC 2146. Clearly more observations need to be undertaken to clarify the nature of this object, and test the validity of this scenario.
Another plausible scenario for this system is that it is at the final stage of merging and the small companion has been completely stripped off of its gas leaving no sign of its nucleus, as suggested by Hutchings et al. (1990) and Lisenfeld et al. (1996). In view of the support that numerical simulations can provide to such mergers (Barnes 1990) and if the above mentioned H I concentration at the south does not provide evidence for a companion nucleus around NGC 2146 this seems also a very attractive possibility.

The NGC 2146 system provides another example to add to those of Hibbard \& van Gorkom (1996) where galaxy-galaxy interactions inject galactic gas into galactic halo regions, as well as ejecting gas with sufficient velocity that it can escape to the intergalactic medium. Depending on the mass ratio of the interacting galaxies, the relative inclinations of their disks and the impact parameter of the encounter the mass lost in the interaction can be up to $60 \%$ of the mass of the companion galaxy (Wallin \& Stuart 1992). This gas contributes to the enrichment of the intergalactic medium in metals and if such events had been very common at some earlier epochs they may help towards finding the objects responsible for the metal absorption-line systems seen in abundance in QSO spectra.

Which of the above scenaria is correct remains yet undetermined. Numerical simulations based on the morphological and kinematical information presented may give us a clearer picture of how the system looked a billion years ago. Nevertheless it is striking that the final result of this collision is a morphologically classified spiral galaxy, although both the galaxy NGC 2146 and its surrounding $\mathrm{HI}$ cloud seem to have contained approximately equal amounts of neutral hydrogen. This system does provide evidence that mergers of two significant systems can be important events in the history of spiral as well as elliptical galaxies.

The encounter will affect the host galaxy for a long time into the future, since the outlying gas residue is likely to fall into the galaxy both from north and south meeting the rotational plane of the galaxy at a significant angle of inclination to its internal orbital plane; this may provide a trickle of missaligned angular momentum for a long time as gas on different trajectories turns around and falls back. In this sense, the effect of this collisional debris will resemble Binney's "Cosmic Drizzle" (1990) as a mechanism for creating long-lived warps in large, isolated galaxies.

\section{Conclusions}

By combining single dish observations and D - configuration VLA data we have produced sensitive, high quality images of the starburst galaxy NGC 2146. These images reveal elongated streams of neutral hydrogen extending out up to 6 Holmberg radii towards both the north and the south of the main galaxy.

The HI morphological and kinematical picture suggests that this galaxy underwent a strong interaction with a LSB companion, which was destroyed during the 
encounter. Although quite disturbed, NGC 2146 retained the morphological characteristics of a spiral galaxy. Analysis of the trajectory of the outlying gas suggests that the interaction was in its most violent phase about $0.810^{9}$ years ago. The infall of the outlying gas to NGC 2146 will continue for a similar timespan preserving a long-lived warp in the disk of this galaxy.

Acknowledgements. We wish to acknowledge the National Astronomy and Ionosphere Center at the Arecibo Observatory for their hospitality during part of the data reduction. The National Radio Astronomy Observatory (NRAO) is operated by Associated Universities, Inc. under cooperative agreement with the National Science Foundation. This research has made use of the NASA/IPAC Extragalactic Database (NED) which is operated by the Jet Propulsion Laboratory, Caltech, under contract with the National Aeronautics ans Space Administration. This work has been supported by National Science Foundation Grant AST 91-19930.

\section{References}

Armus, L., Heckman, T. M., Weaver, K. A., \& Lehnert, M. D. 1995, ApJ, 445, 666

Barnes, J. 1990, IAU Colloq. No. 134

Benvenuti, P., Capaccioli, M., \& Odorico, S. 1975, A\&A, 41, 91

Binney, J. 1990, in Dynamics and Interactions of Galaxies, ed. R. Wielen (Springer Verlag, Berlin), 328

de Blok, W. J. G., McGaugh, S. S., \& van der Hulst, J. M. 1996, MNRAS, 283, 18d
Caswell, J. L., \& Wills, D. 1967, MNRAS, 135, 231

Condon, J. J., Condon, M. A., Gisler, G., \& Puschell, J. J. 1982, ApJ, 252, 102

Della Ceca, R., Griffiths, R. E., Heckman, T. M., Lehnert, M. D., \& Weaver, K. A. 1999, ApJ, 514, 772

Fisher, J. R., \& Tully, R. B. 1975, A\&A, 44, 151

Fisher, J. R., \& Tully, R. B. 1976, A\&A, 53, 397

Gerritsen, J. P. E. 1997, Ph.D. Thesis, University of Groningen Griv, E., \& Chiueh, T. 1998, ApJ, 503, 186

Hibbard, J. E., \& van Gorkom, J. H. 1996, AJ, 111, 655

Hutchings, J. B., Neff, S. G., Stanford, S. A., \& Unger, S. W. 1990, AJ, 100, 60

Jackson, J., \& Ho, P. T. P. 1988, ApJ, 324, L5

Kronberg, P. P., \& Biermann, P. 1981, ApJ, 243, 89

Lisenfeld, U., Alexander, P., Pooley, G. G., \& Wilding, T. 1996, MNRAS, 281, 301

Pease, F. G. 1920, ApJ, 51, 276

Prada, F., Beckman, J. E., Mc Keith, C. D., Castles, J., \& Greve, A. 1994, ApJ, 423, L35

Rao, S., \& Briggs, F. H. 1993, ApJ, 419, 515

de Vaucouleurs, G. 1950, Ann. Astrophys., 13, 362

de Vaucouleurs, G., de Vaucouleurs, A., \& Corwin, H. G. 1976, Second Reference Catalog of Bright Galaxies (University of Texas Press, Austin)

Wallin, J. F., \& Stuart, B. V. 1992, ApJ, 399, 29

Xie, S., Young, J., \& Schloerb, F. P. 1994, ApJ, 421, 434

Young, J. S., Claussen, M. J., Kleinmann, S. G., Rubin, V. C., \& Scoville, N. 1988, ApJ, 331, L81

Zhao, J. H., Anantharamaiah, K. R., Goss, W. M., \& Viallefond, F. 1996, ApJ, 472, 54 